\title{
Some Remarks on Mode and Ray Theories of VLF Radio Propagation
}

\author{
James R. Wait
}

\begin{abstract}
Contribution From the Central Radio Propagation Laboratory, National Bureau of Standards, Boulder, Colo.
\end{abstract}
Some of the assumptions used in treatment of the mode theory of VLF radio propagation are discussed briefly. The connections with geometrical-optical theories are also pointed out.

In several recent papers, the author has discussed waveguide theory of radio propagation for a smooth spherical earth and a concentric anisotropic ionosphere [Wait, 1960; 1963a, 1963b]. To facilitate the solution of this intrinsically difficult problem, certain assumptions were made. In view of great interest in this problem, it appears to be worthwhile to discuss briefly the nature of these assumptions since they have been alluded to in a series of three papers [Berry, 1963; Johler and Berry, 1963; Johler, 1963].

The source is taken to be a vertical electric dipole located at $\theta=0$ and $r=b$ of a spherical coordinate system. The earth's surface is located at $r=a$ and the reference reflecting layer is at $r=c$. The medium between $a$ and $c$ is regarded as free space.

Within certain limitations, which have been discussed extensively [Wait, 1962], the boundary conditions at $r=a$ and $r=c$ may be described in terms of surface impedances. In the general case, the surface impedances are functions of the (complex) angle of incidence. However, for treating VLF propagation in the earth-ionosphere waveguide, it is justified to regard the elements of the surface impedance matrices as constants. As a result, lateraltype waves are neglected. The latter correspond to waveguide modes propagating within the earth and the ionosphere. In the limiting case of a flat earth model, they become part of the continuous spectrum, and they are usually described as the branch-cut waves. A detailed investigation of these lateral waves for a planar model has been carried out by Anderson [1962]. As expected [Wait, 1960], they do not contribute significantly to the total field.

Another consequence of using impedance boundary conditions is that waves "reflected" at the center of the earth are neglected. In essence, this is accomplished by Bremmer $[1949,1958]$ when he replaces the spherical Bessel functions $j_{n}\left(k_{g} r\right)$ by $h_{n}^{(1)}\left(k_{g} r\right)$, where $k_{g}$ is the propagation constant of the earth. In view of the large magnitude of the imaginary part of $k_{g} r$, it turns out that this is an excellent approximation. ${ }^{1}$
Thus, in one fell swoop, the lateral waves and the waves reflected from the center of the earth are dispensed with by the introduction of impedance boundary conditions. Furthermore, in the application of the Watson transform, the desired even property of the integrand is assured [Wait, 1960, 1962].

In much of the theoretical development of this subject, the spherical wave functions $h_{\nu}^{(1)}(x)$ and $h_{\nu}^{(2)}(x)$, of general argument $x$, are ultimately approximated in terms of Airy functions (or Hankel functions of one third). Thus, if $|\nu-x|<<x^{2 / 3}$, it is permissible to write

$$
x h_{\nu}^{(1)}(x) \cong-i(x / 2)^{1 / 6} w_{2}(t),
$$

and

$$
x h_{\nu}^{(2)}(x) \simeq+i(x / 2)^{1 / 6} w_{1}(t)
$$

where

$$
t=\left(\nu+\frac{1}{2}-x\right)(2 / x)^{1 / 3},
$$

and $w_{2}(t)$ and $w_{1}(t)$ are Airy functions. For example

$$
\begin{aligned}
& w_{2}(t)=\sqrt{\pi}[B i(t)+i A i(t)], \\
& w_{1}(t)=\sqrt{\pi}[B i(t)-i A i(t)],
\end{aligned}
$$

in terms of Airy functions $A i(t)$ and $B i(t)$ defined by Miller [1946].

These Airy function representations are highly accurate for the VLF mode problem as has been demonstrated by Wait [1960] and Spies and Wait [1961]. Using such approximations, the modal equation takes on a reasonably tractable form, and numerical results for attenuation and phase velocity may be computed in an economical manner. Despite this, some further approximations are often warranted if simplicity and physical insight are to be gained. For this reason, the Debye approximations for the spherical wave functions are found to be very useful [Wait, 1962]. In the present context, they correspond to utilizing the leading term in the asymptotic expansion of the Airy functions valid for large negative $t$.

The Debye approximated mode equation is accurate when $\left|C_{n}(k a / 2)^{\frac{1}{3}}\right|$ and $\left|C_{n}^{\prime}(k a / 2)^{\frac{1}{3}}\right|>2$, where 
$C_{n}$ and $C_{n}^{\prime}$ are the cosine of the (complex) angle of incidence for the earth and the ionosphere, respectively. Because $(k a / 2)^{\frac{1}{3}}$, at $\mathrm{VLF}$, is the order of 20 and $C_{n}^{\prime}$ is never smaller than about 0.15 , the latter condition is never seriously violated. However, the quantity $\left|C_{n}(k a / 2)^{\frac{1}{3}}\right|$ may become very small at VLF for important modes at frequencies greater than $10 \mathrm{kc} / \mathrm{s}$.

Another approximation occasionally used corresponds to the assumption of a flat earth. Such a model has been used extensively by Budden [1962]. The resulting simplicity permits a careful study of the nature and the properties of the modes. Actually, the flat-earth mode equation is obtained as a limiting case of the spherical-earth equations when both $\left|C_{n}(k a / 2)^{\frac{1}{3}}\right|$ and $\left|C_{n}^{2} \frac{a}{h}\right|>>1$. Extensive comparisons between flat-earth and spherical-earth computations have been reported previously [Wait and Spies, 1960; Wait, 1962].

In certain cases, when the convergence of the sum of waveguide modes is poor, an alternative representation is desirable. Such a representation may be found from geometrical optics wherein the total field is considered to be a sum of rays or hops. The ray or hop series has been obtained directly from the contour-integral representation by Bremmer [1949, 1958]. This result is derived by expanding the integrand, which is of the form $\left[1-R_{g} R_{i}\right]^{-1}$, into a geometrical progression. Individual terms are then evaluated asymptotically to yield the classical geometrical-optical formulas. The method has been extended by Wait [1961] to permit the results to be used in the vicinity of the geometrical horizon of the various rays (i.e., hops). A key point in the development is the ordering of terms so that $R_{g}$ and $R_{i}$ are defined as (spherical) reflection coefficients connecting "downgoing" and "upgoing" wave functions. The latter have the forms $h_{\nu}^{(1)}(k r)$ and $h_{v}^{(2)}(k r)$, respectively, in the earth-ionosphere region. If another choice of wave functions is used (e.g., $j_{\nu}(k r)$ and $\left.h_{\nu}^{(1)}(k r)\right)$, the individual integrals in the expansion should not be identified with geometricaloptical hops in the usual sense. The distinction between these methods of expansion is not trivial. In the first method, the only significant poles are those of the groundwave type, whereas, in the second method, additional poles appear which are closely related to the usual waveguide modes. Thus, if care is not exercised in the choice of wave functions, the individual integrals in the expansion will correspond to hybrid waves which have both guided-wave and ground-wave components. While the resultant field as computed should be identical, the utility of such hybrid expansions could be questioned.

For further discussion of these various points, the reader might wish to consult the referenced literature.

\section{References}

Anderson, W. L. (Jan.-Feb. 1962), Fields of electric dipoles in sea water - the earth-air-ionosphere problem, J. Res. NBS 66D (Radio Prop.), No. 1, 63-72. [Correction in 69D, 63-65, Jan.-Feb. 1963.]

Berry, L. A. (1963), Some remarks on the Watson transformation and mode theory, Digest of Papers, Symposium on the Ionospheric Propagation of VLF Radio Waves, pp. 39-42 (Boulder, Colo., Aug. 12-14).

Bremmer, H. (1949), Terrestrial Radio Waves (Elsevier Publ. Co., New York and Amsterdam).

Bremmer, H. (1958), Propagation of electromagnetic waves, Handbuch der Physik 16, 243.

Budden, K. G. (1962), The Waveguide Mode Theory of Wave Propagation (Prentice-Hall, Englewood Cliffs, N.J.).

Johler, J. R. (1963), Concerning geometrical-optical propagation theory for long waves propagated between the ionosphere and the ground, Digest of Papers, Symposium on the Ionospheric Propagation of VLF Radio Waves, p. 51 (Boulder, Colo., Aug. 12-14).

Johler, J. R., and L. A. Berry (1963), On LF/VLF/ELF terrestrial radio wave fields, Digest of Papers, Symposium on the Ionospheric Propagation of VLF Radio Waves, pp. 43-50 (Boulder, Colo., Aug. 12-14).

Miller, J. C. P. (1946), The Airy Integral (Cambridge University Press).

Spies, K. P., and J. R. Wait (July 17, 1961), Mode calculations for VLF propagation in the earth-ionosphere waveguide, NBS Tech. Note No. 114 (PB-161615).

Wait, J. R. (Mar.-Apr. 1960), Terrestrial propagation of verylow frequency radio waves, J. Res. NBS 64D, No. 2, $153-204$.

Wait, J. R. (June, 1961), A diffraction theory for LF skywave propagation, J. Geophys. Res. 66, No. 6, 1713-1724.

Wait, J. R. (1962), Electromagnetic waves in stratified media (Pergamon Press, Oxford).

Wait, J. R. (May-June 1963a), Concerning solutions of the VLF mode problem for an anisotropic curved ionosphere, J. Res. NBS 6rD (Radio Prop.), No. 3, 297-302.

Wait, J. R. (Feb. 1963b), The mode theory of VLF radio propagation for a spherical earth and a concentric anisotropic ionosphere, Can. J. Phys. 41, 299-315.

Wait, J. R., and K. P. Spies (Aug. 1960), Influence of earth curvature and the terrestrial magnetic field on VLF propagation, J. Geophys. Res. 65, 2325-2331.

(Paper 68D1-319) 